\title{
Obstruction of a Popliteal Artery Stent of a Patient with Popoiteal Artery Entrapment Syndrome
}

\author{
Jae Hyun Kwon \\ Department of Radiology, Dongguk University Ilsan Hospital, Dongguk University, Goyang-si, Republic of Korea \\ Email: jhkwon17@naver.com
}

Received October 28, 2013; revised November 28, 2013; accepted December 5, 2013

Copyright (C) 2013 Jae Hyun Kwon. This is an open access article distributed under the Creative Commons Attribution License, which permits unrestricted use, distribution, and reproduction in any medium, provided the original work is properly cited. In accordance of the Creative Commons Attribution License all Copyrights (c) 2013 are reserved for SCIRP and the owner of the intellectual property Jae Hyun Kwon. All Copyright (C) 2013 are guarded by law and by SCIRP as a guardian.

\begin{abstract}
We encountered a popliteal artery entrapment syndrome (PAES) patient who was treated with stent placement for popliteal artery without correcting an abnormal muscular structure that compresses popliteal artery in another institution. The popliteal artery stent was occluded one year after the stent placement. The patient was treated with bypass graft by reversed saphenous vein. Interventionists should be cautious with stent placements for popliteal artery without adjusting the anomalous musculotendinous structure in patients with PAES.
\end{abstract}

Keywords: Popliteal Artery Entrapment Syndrome; Autogenous Saphenous Vein Graft; Treatment; Stent Placement; Endovascular

\section{Introduction}

Popliteal artery entrapment syndrome (PAES) is an anomalous relationship between the popliteal artery and its surrounding musculotendinous structures [1]. Selected treatment of PAES is a surgical method which includes the division of an anomalous musculotendinous structure and a vascular reconstruction [1-3]. Percutaneous thrombolysis or stent placement has not been fully investigated in managing PAES [2,4-6]. We report a patient with PAES in which popliteal artery stent eventually resulted in occlusion and was treated for surgical bypass.

\section{Case Report}

A 46-year-old man without atherosclerotic risk factor was admitted with right calf claudication after walking $100 \mathrm{~m}$ for the four-month duration prior to being admitted. The ankle-brachial index (ABI) of the lower right extremity was unrecognized. During the physical examination, the right dorsalis pedis and posterior tibial arteries showed very weak pulse. ABI of the lower left extremity was normal (0.9). The patient had been diagnosed as diabetes mellitus and has been taking medication for seven years. He has smoked one and half pack/day for 20 years. A year ago, before this incident, he was faced with sudden extreme pains on his lower right. The patient was misdiagnosed as atherosclerotic occlusion of the right popliteal artery at another institute. The occluded right popliteal artery was percutaneously recanalized and a stent placement was performed without any treatment of the anomalous musculotendinous structure of the lower right extremity. The patient was discharged after the stent placement for the occluded right popliteal artery and faced no further symptoms for one year.

At our hospital, he underwent a multi-detector computed tomographic angiography (CTA). A 64-slice MDCT scanner was used for scanning (Somatom Sensation 64; Siemens, Forchheim, Germany) with about 150 $\mathrm{cm}^{3}$ of contrast medium (Ultravist; Bayer Shering Pharma AG, Berlin, Germany) in a rate of $4 \mathrm{~cm}^{3} / \mathrm{sec}$. CTA displayed the occlusion of the right popliteal artery and stent previously inserted at the other hospital. CTA demonstrated an entrapment of the right popliteal artery and stent by an anomalous muscle band of medial head of the right gastrocnemius muscle consistent with Type III PAES [7] (Figure 1). Abdominal aorta, iliac, femoral and crural vessels of both lower right and left extremities showed no stenotic or obstructive lesion. Left knee showed normal relationship between popliteal vessels and gastrocnemius muscle. And also left popliteal artery showed good patency and contrast enhancement. The patient was diagnosed as PAES of the right knee which 

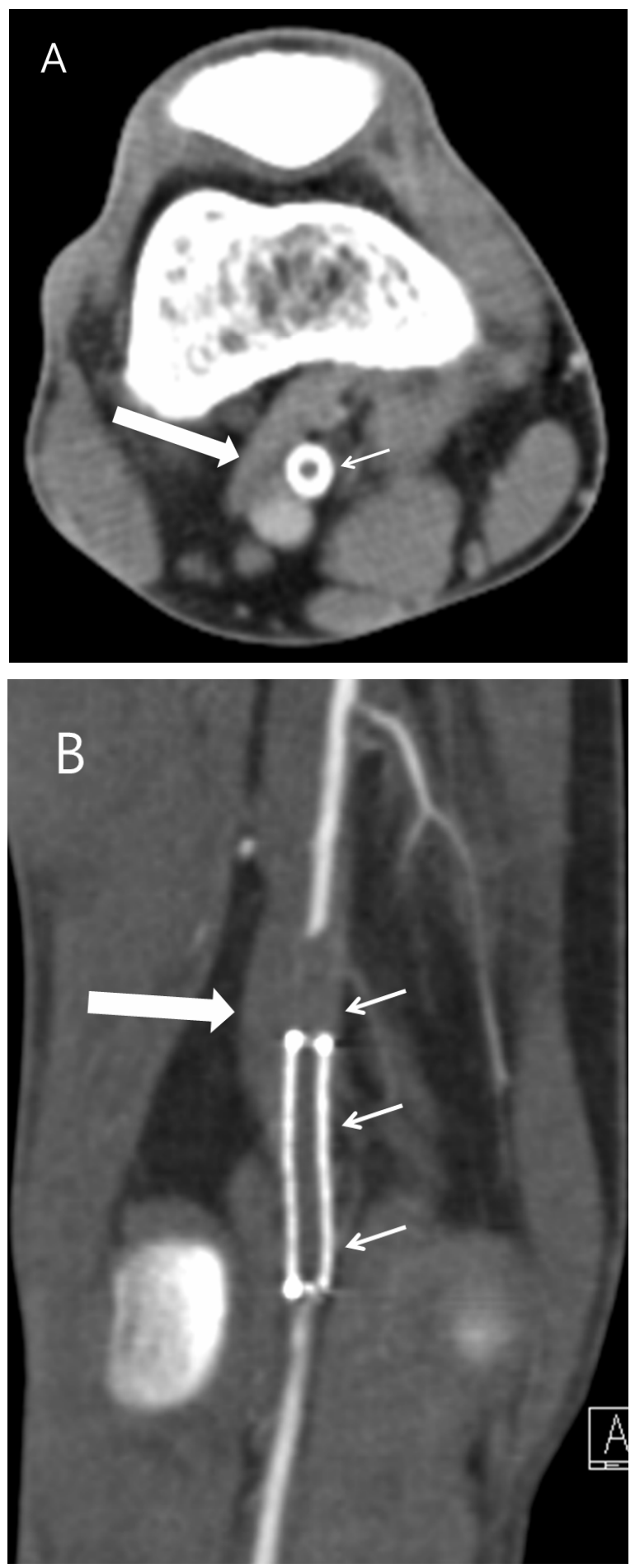

Figure 1. Right knee of a 46-year-old male with popliteal artery entrapment syndrome. (A) Axial image of CTA of the right knee reveals that the occluded right popliteal artery stent (short arrow) is entrapped by an anomalous band (long arrow) of medial head of the right gastrocnemius muscle; (B) Coronal reformatted image of the right knee demonstrates that obstruction of the right popliteal artery and the stent (short arrows) is entrapped by an anomalous band of medial head of the right gastrocnemius muscle (long arrow). resulted in the occlusion of the right popliteal artery and stent. The patient underwent surgical treatment. Through a medial incision, the popliteal artery was bypassed using the reversed ipsilateral great saphenous vein, with the proximal anastomosis on the distal part of the superficial femoral artery and the distal anastomosis on the distal popliteal artery. The patient recovered accordingly and was discharged with normal ABI (0.93) of lower right extremity. Twenty-four months after the bypass surgery, the patient was asymptomatic with ABI of 0.92 at lower right extremity. Confirm that you have the correct template for your paper size. This template has been tailored for output on the custom paper size $(21 \mathrm{~cm} * 28.5 \mathrm{~cm})$.

\section{Discussion}

PAES is caused by an anomalous musculotendinous relationship that externally compresses the popliteal artery [8]. Popliteal artery entrapment syndrome is an uncommon clinical entity. The incidence of the PAES has been reported to range from $0.16 \%$ to $3.5 \%$ in a study by healthy volunteers and a study by the autopsy specimens $[3,9]$. PAES usually occurs in young athletic male patients who lack the risk factors for atherosclerosis and who are physically healthier $[1,9]$. The most common presenting symptom is progressive claudication during sports or exercise [1]. Rarely, resting pain or necrosis of the limb can be the initial presenting symptom [8]. The treatment of PAES of an early stage is indispensable because PAES is a progressive disease and finally popliteal artery may result in occlusion [9]. Bilateral popliteal artery involvement has been reported from $22 \%$ to $67 \%$ of presenting cases [7]. In our patient's case, the right popliteal artery was involved, but left popliteal fossa showed normal relationships between popliteal artery and medial head of gastrocnemius muscle.

CTA shows detailed images not only in the lumen and the wall of the popliteal artery, but also in the presence of aberrant muscle, the abnormal relationship between the popliteal artery and surrounding structures [7,9]. CTA may be the preferred procedure for young adults with vascular symptom in lower extremities [9]. In our case, CTA revealed the occlusion of the popliteal artery and stent and well depicted the relationship between an anomalous muscular band of the medial head from the right gastrocnemius muscle and popliteal artery.

If it is untreated, the compression mechanism will frequently result in deterioration of the popliteal artery that may eventually lead to occlusion [9]. Popliteal artery entrapment syndrome must be treated by surgery. Surgical treatment technique is the releasing of vessel by extracting the muscle that causes entrapment, and reconstructing the narrowed lumen by endarterectomy or bypass graft [9]. Relief of the constricting lesion with musculotendinous section will suffice for an entrapped pop- 
liteal artery with normal findings [3]. Long-term follow-up data of the patients treated by surgical methods are very restricted [6]. Di Marzo et al. reported a patency rate of the popliteal artery of $94 \%$ after a mean follow-up of 46 months in 11 PAES patients without vascular complications treated by only division of anomalous musculotendinous structures [2]. The patency rate was 58\% after a mean follow-up of 43 months in 12 PAES patients needed vascular reconstruction due to arterial complications [2].

Endovascular treatment, such as percutaneous catheter-directed thrombolysis with or without balloon angioplasty, is not effective without removing the underlying reason of vessel entrapment, where the risk of re-occlusion is high $[6,8,9]$. Marzo et al. reported a case with popliteal artery entrapment syndrome in which popliteal stent was occluded. The patient underwent arterial reconstruction and resection of the medial gastrocnemius muscle after two unsuccessful treatments with arterial recanalization and stenting [4]. Burger et al. reported a case in which stent-graft was placed for PAES and concluded that durability of the stent-graft will require longer follow-up times and evaluations in a greater number of patients [5]. Steurer et al. reported three cases in which percutaneous thrombolysis, thromboembolectomy and balloon angioplasty was performed for occlusion of popliteal artery due to PAES [6]. However, these three patients have undergone surgical correction of the underlying musculotendinous abnormality three to six weeks after the percutaneous treatment. They concluded that preserving the native popliteal artery may result in a better prognosis, but, long-term results in respect to aneurysm formation and vessel patency have yet to be evaluated. We realize that in the treatment of PAES patient, percutaneous treatments such as thrombolysis or thromboembolectomy without correction of underlying anomalous musculotendinous structure has limitations. And also percutaneous stent or stent graft treatment for occluded popliteal artery should be evaluated for long-term patency in larger study populations.

\section{Conclusion}

In patients with PAES, primary treatment goal is releasing the musculotendinous structures that cause entrapment of the popliteal artery. Percutaneous primary stenting of the occluded popliteal artery without correcting underlying abnormal structure that compresses and irritates popliteal artery may result in obstruction of the stent. Interventionist should be cautious about stent placement at popliteal artery occlusion in patients with PAES and also PAES must be considered as the cause of the popliteal artery occlusion in young healthy patients even those who have atherosclerotic risk factors.

\section{Acknowledgements}

None Declared.

\section{REFERENCES}

[1] B. K. Goh, K. H. Tay and S. G. Tan, "Diagnosis and Surgical Management of Popliteal Artery Entrapment Syndrome,” ANZ Journal of Surgery, Vol. 75, No. 10, 2005, pp. 869-873. http://dx.doi.org/10.1111/j.1445-2197.2005.03555.x

[2] L. di Marzo, A. Cavallaro, V. Sciacca, A. Mingoli and A. Tamburelli, "Surgical Treatment of Popliteal Artery Entrapment Syndrome: A Ten-Year Experience,” European Journal of Vascular and Endovascular Surgery, Vol. 5, No. 1, 1991, pp. 59-64. http://dx.doi.org/10.1016/S0950-821X(05)80928-4

[3] N. Ohara, T. Miyata, H. Oshiro and H. Shigematsu, "Surgical Treatment for Popliteal Artery Entrapment Syndrome," Cardiovascular Surgery, Vol. 9, No. 2, 2001, pp. 141-144. http://dx.doi.org/10.1016/S0967-2109(00)00110-1

[4] L. di Marzo, A. Cavallaro, S. D. O’Donnell, H. Shigematsu, L. J. Levien and N. M. Rich and Popliteal Vascular Entrapment Forum, "Endovascular Stenting for Popliteal Vascular Entrapment Is Not Recommended,” Annals of Vascular Surgery, Vol. 24, No. 8, 2010, pp. 1131-1133.

[5] T. Burger, F. Meyer, J. Tautenhahn, Z. Halloul and J. Fahlke, "Initial Experiences with Percutaneous Endovascular Repair of Popliteal Artery Lesions Using a New PTFE Stent-Graft," Journal of Endovascular Surgery, Vol. 5, No. 4, 1998, pp. 365-372.

http://dx.doi.org/10.1583/1074-6218(1998)005<0365:IE WPER $>2.0 . \mathrm{CO} ; 2$

[6] J. Steurer, U. Hoffmann, E. Schneider, J. Largiader and A. Bollinger, "A New Therapeutic Approach to Popliteal Artery Entrapment Syndrome (PAES),” European Journal of Vascular and Endovascular Surgery, Vol. 10, No. 2, 1995, pp. 243-247. http://dx.doi.org/10.1016/S1078-5884(05)80120-0

[7] H. Zhong, C. Liu and G. Shao, “Computed Tomographic Angiography and Digital Subtraction Angiography Findings in Popliteal Artery Entrapment Syndrome,” Journal of Computer Assisted Tomography, Vol. 34, No. 2, 2010, pp. 254-259.

http://dx.doi.org/10.1097/RCT.0b013e3181bbf173

[8] G. Zund and U. Brunner, "Surgical Aspects of Popliteal Artery Entrapment Syndrome: 26 Years of Experience with 26 Legs,” Vasa, Vol. 24, No. 1, 1995, pp. 29-33.

[9] S. Papaioannou, K. Tsitouridis, G. Giataganas, G. Rodokalakis, V. Kyriakou, C. Papastergiou, M. Arvaniti and I. Tsitouridis, "Evaluation of Popliteal Arteries with CT Angiography in Popliteal Artery Entrapment Syndrome," Hippokratia, Vol. 13, No. 1, 2009, pp. 32-37. 